\title{
End-of-life matters in chronic heart failure patients
}

Piotr Sobanski, Tiny Jaarsma and Malgorzata Krajnik

\author{
Linköping University Post Print
}

\section{Tweet}

N.B.: When citing this work, cite the original article.

Original Publication:

Piotr Sobanski, Tiny Jaarsma and Malgorzata Krajnik, End-of-life matters in chronic heart failure patients, 2014, CURRENT OPINION IN SUPPORTIVE AND PALLIATIVE CARE, (8), 4, 364-370.

http://dx.doi.org/10.1097/SPC.0000000000000094

Copyright: Lippincott, Williams \& Wilkins

http://www.lww.com/

Postprint available at: Linköping University Electronic Press

http://urn.kb.se/resolve?urn=urn:nbn:se:liu:diva-112803 
End-of-life matters in chronic heart failure patients

Piotr Sobanski ${ }^{1,2}{ }^{*}$, Tiny Jaarsma ${ }^{3}$, Malgorzata Krajnik ${ }^{4}$

1. Hildegard Palliative Centre Basel, Switzerland

2. Gynaecological Cancer Centre, University Hospital Basel, Switzerland

3. Department of Social and Welfare Studies, Linköping University, Sweden

4. Department of Palliative Care, Nicolaus Copernicus University, Collegium

Medicum in Bydgoszcz, Poland

Correspondence to:

Piotr Sobanski, MD, PhD

Palliativzentrum Hildegard, St. Alban-Ring 151, 4052 Basel, Switzerland

Tel. 0041613197575

Fax. 0041613197559

\begin{abstract}
Purpose of review

Until recently, concepts of care for people with heart failure (HF) had rarely included preparation for unavoidable imminent death or caring for the dying.

The purpose of this review is to provide an update on current end-of-life (EoL) issues specific to HF patients.
\end{abstract}

\title{
Recent findings
}

Mortality in the HF population remains high, especially short after the first acute HF hospitalisation. Patients with systolic HF die more frequently from progressive HF or sudden cardiac death (SCD); patients with diastolic HF for non-cardiovascular reasons and SCD. The mode of haemodynamic decline leading to HF death can be characterised by low cardiac output with or without secondary end-organ dysfunction, congestion or a combination of both. A new model of EoL trajectories has been proposed which takes into account this influence on the prognosis of HF and comorbidities. Advance care planning for patients with implanted cardiac devices has been shown to be unsatisfactory. A recent strategy for managing implantable cardioverter defibrillators in patients approaching death is presented.

\section{Summary}

There is an emerging need to define specific challenges for EoL care for approaching death in HF patients. More research and education are needed to improve care for dying HF patients, including those with implanted cardiac devices.

Keywords: heart failure, end of life, death and dying, implanted cardiac devices 


\section{Introduction}

Heart failure (HF) is principally an incurable and progressive disease. Thus, once diagnosed, it will be present in a person's life until death caused by HF itself, a concomitant disease or another reason, such as an accident. Sudden cardiac death (SCD) can confound any scenario at any point. Increasing numbers of people with HF have had different cardiac devices implanted, improving quality of life and/or survival. Eventually, at end of life (EoL), when death becomes imminent, these devices preventing death may prolong dying, rather than life. For this reason, the modification of the activity of these devices should be considered and discussed with the patient in advance.

The purpose of this paper is to review current EoL issues in HF patients. Symptom control, however crucially important, will not be discussed due to the limited scope of this paper.

\section{Mortality}

Despite progress in management, the mortality for HF, although improving, remains high. Three-year mortality is about 25\%.[1] The highest mortality is observed shortly after the first acute HF hospitalisation (10-20\% die within one month).[2] The latter prognosis seems to be better if only the patients who survive this vulnerable period are taken into account, after those with "malignant disease" have already died.[3] The average life expectancy after the first episode of HF is 2.34 years in men and 1.79 years in women, and 1.1 year after considering patients as eligible for heart transplantation and 9.4 months for those who are too ill to be put on the waiting list. $[4,5]$

\section{Causes of death}

The causes of death can be categorised as cardiac, non-cardiac and sudden. Cardiac deaths can be subdivided into tachyarrhythmic, bradyarrhythmic, progressive HF, non-arrhythmic and non-HF, and cardiac without the possibility of further subspecification.[6] In the HF population in recent decades, the contribution of SCD to general mortality has been decreasing; non-cardiovascular mortality has been rising, and progressive HF remains stable.[7] Rates of SCD have improved due to both medical treatment (beta-blockers) decreasing the risk of malignant arrhythmia 
and devices (implantable cardioverter defibrillators [ICDs] or cardiac resynchronisation therapy [CRT] with defibrillating function [CRT-D]) terminating arrhythmia. It was supposed that the implantation of ICDs would shift the reasons for mortality towards progressive heart failure, but this has not been observed.[6] People saved from SCD tend to die for non-HF-related reasons. One possible explanation for this shift is the aging of both the general and HF populations. Multi-morbidity and incidence of HF rise with age, so the average HF patient suffers from more than four co-morbidities. The HF population can be divided into two groups: those with impaired systolic function of the left ventricle (systolic HF, or HF with reduced ejection fraction [HFREF]) and those with impaired filling of the left ventricle (diastolic HF or HF with preserved ejection fraction [HFPEF].[8, 9] The distribution of causes of death is related to the type of HF (Table 1). The general mortality is similar in both groups (Figure 1). People with HFREF die more frequently from cardiovascular causes (HF and SCD), and those with HFPEF from non-cardiovascular ones and SCD. If only cardiovascular deaths are considered, the proportion of SCD becomes comparable for both groups, although HF deaths are still more common in systolic HF. The main form of cardiovascular death is progressive HF in HFREF and SCD in HFPEF. The rate of non-cardiovascular deaths in HFPEF rises proportionally with age. The most common concomitant diseases leading to death in HFPEF are cancer and infection. $[8,10,11]$

\section{Modes of HF death}

Dying from progressive HF can be characterised by low cardiac output (CO) with or without secondary end-organ dysfunction, congestion or a combination of both. The event directly terminating life can be bradyarrhythmia, tachyarrhythmia (ventricular fibrillation) or pulseless rhythm (electromechanical dissociation). The most common form of HF death in HFREF is low CO, alternatively with congestion, whereas in HFPEF it is congestion alternatively with low CO.[12] In both forms of HF, CO and stroke volume (SV) can be reduced to a similar degree (Table 2). In systolic HF the volume of blood pumped out with one contraction (SV) corresponding with 10-15\% (EF) of left ventricle end diastolic volume (LVEDV) can be equal with 50\% (EF) of small LVEDV typically seen in HFPEF. If low CO leads to secondary dysfunction of other organs, patients will present additional symptoms of renal or liver failure, 
impaired digestion or somnolence. If local factors (such as atherosclerotic plaques) further compromise flow, further deterioration of organ function up to necrosis is seen (intestinal infarction, limb necrosis or stroke). Sometimes, additional thromboembolic incidents may complicate the above scenarios. Decreased CO activates the neurohormonal axis and leads to salt and water conservation, vasoconstriction and elevation of ventricular filling pressure, with subsequent congestion in pulmonary or systemic circulation.[8]

\section{Dying trajectories}

Death from HF is usually seen as unpredictable, with about $50 \%$ of deaths being sudden. The Seattle Heart Failure Model (SHFM) does not seem to be the optimal assessment tool for the stratification of risk of death respective planning EoL care. Using the SHFM, only $0.4 \%$ of HF patients would be classified as very high risk (one-year mortality of $84 \%$ ) and $3.3 \%$ as intermediate risk (mortality > 50\%).[13] A recent retrospective analysis investigating the pattern of how HF patients approached death showed that only $21 \%$ of deaths were sudden. The authors identified five EoL trajectories (Figure 2): 1. Constant moderate risk of death without deterioration before death (unexpected SCD); 2. Early (within 12 months); 3. Late or 4. Rapid (up to 3 months) progression to high probability of death; and 5. Constant high risk of death. This model provides a more accurate prediction of death because it respects both HFand co-morbidity-related factors.[14] However, modern model of implementing palliative care (PC) for people with HF is no longer based on risk-of-death prediction, but on recognition of PC needs. PC services should be integrated with cardiological ones along the whole disease trajectory ("parallel care”). Prognostication is not needed to implement PC, but can be valuable for planning EoL care, which nowadays is usually organised ad hoc.

\section{Need for better communication}

Medical staff are convinced their communication with patients is excellent. In the patients' opinion, however, communication is satisfactory only in respect to everyday care, but not EoL issues. Using the Quality of Communication questionnaire, patients assessed ( 0 = worst; 10 = best) the communication with the treating physician on care as having a median score of 8.0 [7.0-8.7] but on EoL with a median score of 0.0 [0.0- 
1.3].[15] Advance directives (AD), referring to preferences regarding possible needs for therapeutic interventions such as cardiopulmonary resuscitation or mechanical ventilation, should be discussed with patients and their families and the validity of the orders checked regularly. In addition to general issues, disease-specific matters, such as those related to implantable devices (both electronic and mechanical) should be addressed as well. Evidence shows that most patients with implanted ICDs are not aware of the possibility of deactivating the device. This is not consistent with recent efforts to move conversations about ICD deactivation to a time prior to implantation. Interestingly, ADs still play a limited role, being prepared only by $41 \%$ of HF patients in the US and 3.9\% in Netherlands.[15, 16] The presence of ADs decreases the intensity of treatment (reduction in ICU hospitalisations and mechanical ventilation), but not the frequency of hospital admissions.

For good decision making, patients need precise information about the disease, prognosis, possible interventions and the probabilities of their positive and negative outcomes. Patients receive scant information concerning the probability of success of potential resuscitation or ICD intervention. They perceive resuscitation as noninvasive intervention, having a high probability of success. Patients imagine, that ICDs save 50 lives per 100 patients over five years. In fact, absolute risk of death reduction is only $5-7 \%$ during $3-5$ years (this corresponds to $23-36 \%$ relative risk reduction).[17, 18] The prognosis is only rarely discussed with patients because many physicians think it is too unpredictable and will diminish hope.[19] Of course, not every patient expects such discussion and this has to be respected. However, the principle of "being prepared for the worst while hoping for the best" leaves a big space in which to recognise the need for conversation.[20]

As the disease progresses, the goals of treatment should be re-evaluated, as previous aims can become unrealistic and reflect false hope. Attempts to achieve them can lead to medical futility. As a consequence, the imminently dying are treated at full intensity with the attitude of implementing anything possible. The content of communication should be adapted to the stage of advancement of the disease. In the less-advanced stages, it is more important to discuss the risk of sudden death, whereas in later disease progression, slow decline and low cardiac output trajectory become more necessary to discuss. The treatment preferences of patients can never be assumed automatically. It is generally believed that elderly persons will prioritise 
quality over length of life. Studies show, however, that the vast majority (74\%) of elderly people with HF are not willing to trade-off survival time for quality of life. [21]

\section{Approaching death with active implantable devices}

Implantable cardiological devices can be electronic (cardiovascular implantable electronic devices [CIEDs]) or mechanical (ventricular assist devices [VADs]). They improve or support functioning of the heart (CRT or VADs) or decrease the risk of SCD (ICDs). ICDs can terminate potentially fatal arrhythmia using two different patterns of intervention, depending on the type of arrhythmia and programming: highenergy electrical shock (usually painful) or low-energy antitachycardia pacing (not noticeable). As patients approach death from a cause that is not amenable to a device, some of their functions (VADs and shocks from ICDs, or CRT-D), if left unadjusted, can worsen the dying scenario. The shock does not treat the underlying condition, but simply terminates arrhythmia. If arrhythmia is an element of terminal agony, the device is attempting to "resuscitate" the dying person and returns him or her to a state of agony. In such conditions, an ICD no longer provides holistically considered benefits. The deactivation of an ICD allows a natural death that is unavoidable and imminent. However, discussion on this topic is not easy, either for the patient and/or his or her representative or for the physician. Patients make the decision more intuitively than rationally, even if they seem to understand the role of an ICD. Asked about deactivating their ICD, they usually answer "no", because the ICD prevents SCD, which is intuitively understood as preventing any death. The same person asked about preferring a slow death, however, usually answers "no" as well. These answers are contradictory and reflect misunderstanding of the essence of the two questions.[22] This shows how important is pattern of the communication.

In decision making regarding modification of CIED activity, every kind of intervention, having various consequences, needs separate consideration even if delivered by one device. Pacing (which can be delivered by pacemakers, CRT and ICDs) per se does not have to interfere with the dying process, because the sensitivity of the heart is falling, and the probability of effective stimulation decreases along with the changes in homeostasis typically seen during dying (such as acidosis or dyselectrolitaemia). The rising stimulation threshold makes the heart less susceptible 
to pacing, thus evoking prolonged dying is less likely. CRT is a special type of stimulation, restoring the synchrony of left ventricle contraction and improving the efficacy of the work of the heart. Both forms of pacing - antibradycardia and resynchronisation - prevent symptoms, and do not cause any suffering during dying. Loss of function can itself be a cause of suffering: syncope in the case of the discontinuation of antibradycardia pacing and a escalation of HF symptoms caused by loss of resynchronisation.[23]

The modification of high- (shock) and low-voltage (anti-tachycardia pacing) interventions of ICD and CRT-D needs separate consideration. It is imaginable that one would like to avoid painful interventions and keep anti-tachycardia pacing active. This allows the patient to possess a device that is not useless, while being protected from device-induced suffering. Of patients with a fully active device, "only” $21 \%$ will receive shocks whilst dying.[17] Other terminal events - bradycardia or pulseless rhythm - do not trigger ICD intervention. It had been anticipated that HF patients approaching EoL would wish to modify ICD function quite often. Available data are quite divergent and show that $30-71 \%$ of questioned HF patients can imagine requesting to switch off an ICD in at least one clinical scenario of imminent death or suffering.[18, 24, 25] It is hypothesised that the exceptionally high proportion of patients potentially expecting ICD deactivation (71\%) presented in one study was the result of scheduled education being an obligatory part of the decision-making process.[24] The National Quality Forum in the US has proposed using deactivation of ICDs as a quality indicator of PC for hospitalised persons with an anticipated death (http://www.qualityforum.org/Projects/Palliative_Care_and_End-of-Life_Care.aspx

accessed August, 2014). The proposed quality factor is the proportion of dying patients that have an ICD deactivated or where the reasons for not doing so have been documented. A project was developed to improve quality regarding ICDs in hospice inpatients and in home care based on three elements: education, documentation and clinical tools. If deactivation was not obtained in the preferred electronic way, the usage of magnets was available. Placing a magnet on the device could be performed on a patient's verbal instruction. To make this possible, every clinician treating patients was equipped with ICD-deactivation magnets, and these were also left in patients' homes to prevent repeated shocks in the case of an emergency. These three interventions (education, documentation, and clinical tools) and the presence of the 
policy allowed an almost four-fold increase in the frequency of dying with a deactivated ICD.[26]

Another type of device that changes the trajectory for HF patients is a VAD, primarily used as a bridge to transplantation but being used more often as a long-term destination therapy. The issue was discussed in detail recently by one of the coauthors (TJ).[27] Decisions on discontinuing VADs are complex, as the device replaces native physiological heart function in HF and its deactivation is a life-ending intervention. Rady and Verheijde postulate that the onset of a new lethal pathophysiology (i.e., circulatory shock, massive haemorrhage, overwhelming infection, multiple organ failure, or terminal cancer) is a key criterion in assessing the permissibility of the lifeending discontinuation of a VAD and in differentiating natural death from assisted death.[28] Medicare and Medicaid in the US have stipulated that centres implanting VADs as a destination therapy include a PC specialist as a member of the team, due to the fact that durable mechanical circulatory support might significantly alter EoL trajectory (www.cms.gov/medicare-coverage-database/details/nca-proposed-decisionmemo.aspx?NCAId=268).

\section{Conclusion}

The awareness that HF can progress with specific but diverse trajectories is increasing. Dying is, of course, an integral part of each trajectory. As modern drug and device therapies influence the whole disease trajectory, they can significantly influence the quality of dying. A decreasing proportion of HF patients is dying from SCD, raising the number of people living with HF who will approach death due to other, non-cardiological reasons. A large proportion of the latter will have active implantable cardiac devices in place during the phase of dying. Support for people dying from or with HF can and should require the re-evaluation of treatment goals, symptom management. Additionally patients having the implantable devices modification of their activities should be disused (preferentially in advance) and if expected performed at appropriate time. To enable good EoL care, the participation of patients in a decision-making process based on good communication is crucial. In an attempt to identify unmet needs of patients and their relatives and to develop more effective modes of caring for them, the new Task Force on Palliative Care for People 
with Heart Disease was created in 2014 under the auspices of the European Association for Palliative Care. [29]

\section{Word count 2627}

\section{Key points:}

1. Patients with advanced HF die from cardiovascular and a growing proportion of non-cardiovascular causes.

2. There is a need for better anticipatory communication about possible disease trajectories.

3. Modern management (in the first instance implantable devices) saves life but eventually influences scenarios for dying. Prompt advance care planning is recommended.

4. Technology allows postponing death, sometimes at the cost of a good quality of life. The possibility of influencing the length of a very limited life involves ethical dilemmas.

\section{Most relevant publications}

$1{ }^{*}$ Most recent survival meta-analysis presenting data from 31 studies embracing 41949 patients with HF.

$14 * *$ The new risk of death prediction model. Better prognostic accuracy is based on respecting both HF- and co-morbidity-related factors. Previously models were based exclusively on HF-risk factors.

$23 * *$ Overview of end-of-life issues related to pacemakers.

$26 *$ Analysis of factors improving implementation of ICD deactivation among patients near EoL.

27* Overview of care and communication respective EoL in patients with VAD as destination therapy.

28** Paper presenting ethical complexity of EoL decision-making in patients with VADs.

29** Paper presenting current project dedicated to improvement of PC for people with heart disease, in the first instance HF 


\section{References:}

1. Martinez-Selles M, Doughty RN, Poppe K, et al. Gender and survival in patients with heart failure: interactions with diabetes and aetiology. Results from the MAGGIC individual patient meta-analysis. Eur J Heart Fail. 2012;14(5):473-479.

2. Chang PP, Chambless LE, Shahar E, et al. Incidence and survival of hospitalized acute decompensated heart failure in four US communities (from the Atherosclerosis Risk in Communities Study). Am J Cardiol. 2014;113(3):504510.

3. Frohlich GM, Holzmeister J, Hubler M, et al. Prophylactic implantable cardioverter defibrillator treatment in patients with end-stage heart failure awaiting heart transplantation. Heart. 2013;99(16):1158-1165.

4. Jhund PS, Macintyre K, Simpson CR, et al. Long-term trends in first hospitalization for heart failure and subsequent survival between 1986 and 2003: a population study of 5.1 million people. Circulation. 2009;119(4):515523.

5. Long E, Swain G, Mangi A. Comparative survival and cost-effectiveness of advanced therapies for end-stage heart failure. Circulation Heart failure. 2014;7(3):470-478.

6. Thijssen J, van Rees JB, Venlet J, et al. The mode of death in implantable cardioverter-defibrillator and cardiac resynchronization therapy with defibrillator patients: Results from routine clinical practice. Heart rhythm. 2012;9(10):1605-1612.

7. Cubbon RM, Gale CP, Kearney LC, et al. Changing characteristics and mode of death associated with chronic heart failure caused by left ventricular systolic dysfunction: a study across therapeutic eras. Circ Heart Fail. 2011;4(4):396-403. 8. Zile MR, Gaasch WH, Anand IS, et al. Mode of death in patients with heart failure and a preserved ejection fraction: results from the Irbesartan in Heart Failure With Preserved Ejection Fraction Study (I-Preserve) trial. Circulation. 2010;121(12):1393-1405.

9. Rickenbacher P, Pfisterer M, Burkard T, et al. Why and how do elderly patients with heart failure die? Insights from the TIME-CHF study. Eur J Heart Fail. 2012;14(11):1218-1229.

10. Rutten FH, Heddema WS, Daggelders GJ, et al. Primary care patients with heart failure in the last year of their life. Family practice. 2012;29(1):36-42.

11. O'Connor CM, Miller AB, Blair JE, et al. Causes of death and rehospitalization in patients hospitalized with worsening heart failure and reduced left ventricular ejection fraction: results from Efficacy of Vasopressin Antagonism in Heart Failure Outcome Study with Tolvaptan (EVEREST) program. Am Heart J. 2010;159(5):841-849 e841.

12. Hamaguchi S, Kinugawa S, Sobirin MA, et al. Mode of death in patients with heart failure and reduced vs. preserved ejection fraction: report from the registry of hospitalized heart failure patients. Circulation journal : official journal of the Japanese Circulation Society. 2012;76(7):1662-1669. 
13. Kalogeropoulos AP, Georgiopoulou VV, Giamouzis G, et al. Utility of the Seattle Heart Failure Model in patients with advanced heart failure. J Am Coll Cardiol. 2009;53(4):334-342.

14. Kheirbek R, Alemi F, Citron B, et al. Trajectory of illness for patients with congestive heart failure. Journal of palliative medicine. 2013;16(5):478-484.

15. Janssen DJ, Spruit MA, Schols JM, et al. A call for high-quality advance care planning in outpatients with severe COPD or chronic heart failure. Chest. 2011;139(5):1081-1088.

16. Dunlay SM, Swetz KM, Mueller PS, et al. Advance directives in community patients with heart failure. Circ Cardiovasc Qual Outcomes. 2012;5(3):283-289.

17. Matlock DD, Stevenson LW. Life-saving devices reach the end of life with heart failure. Prog Cardiovasc Dis. 2012;55(3):274-281.

18. Stewart GC, Weintraub JR, Pratibhu PP, et al. Patient expectations from implantable defibrillators to prevent death in heart failure. J Card Fail. 2010;16(2):106-113.

19. Dunlay SM, Roger VL. Living and dying with heart failure: it's time to talk. Eur Heart J. 2012;33(6):689-691.

20. Barclay S, Momen N, Case-Upton S, et al. End-of-life care conversations with heart failure patients: a systematic literature review and narrative synthesis. The British journal of general practice : the journal of the Royal College of General Practitioners. 2011;61(582):e49-62.

21. Brunner-La Rocca HP, Rickenbacher P, Muzzarelli S, et al. End-of-life preferences of elderly patients with chronic heart failure. Eur Heart J. 2012;33(6):752-759.

22. Matlock DD, Allen LA. Defibrillators, deactivation, decisions, and dying. JAMA internal medicine. 2013;173(5):375-394.

23. Reith S, Janssens U. [Dying with/despite a pacemaker]. Medizinische Klinik - Intensivmedizin und Notfallmedizin. 2014;109(1):19-26.

24. Dodson JA, Fried TR, Van Ness PH, et al. Patient preferences for deactivation of implantable cardioverter-defibrillators. JAMA internal medicine. 2013;173(5):377-379.

25. Herman D, Stros P, Curila K, et al. Deactivation of implantable cardioverter-defibrillators: results of patient surveys. Europace.

2013;15(7):963-969.

26. Kraynik SE, Casarett DJ, Corcoran AM. Implantable Cardioverter Defibrillator Deactivation: A Hospice Quality Improvement Initiative. J Pain Symptom Manage. 2014;48(3):471-477.

27. Ben Gal T, Jaarsma T. Self-care and communication issues at the end of life of recipients of a left-ventricular assist device as destination therapy. Curr Opin Support Palliat Care. 2013;7(1):29-35.

28. Rady MY, Verheijde JL. Ethical challenges with deactivation of durable mechanical circulatory support at the end of life: left ventricular assist devices and total artificial hearts. Journal of intensive care medicine. 2014;29(1):3-12. 29. Sobanski P, Janssen DJ, Hogg KJ, et al. EAPC Task Force on Palliative Care for People with Heart Disease: rationale, aims and proposed outcomes. BMJ Supportive \& Palliative Care.Submitted. 


\section{Acknowledgements}

Funding sources: none

\section{Conflicts of interest}

The authors declare that they do not have any potential conflicts of interest directly relating to the work presented in this article. All the authors have seen, reviewed, and approved this article. All the authors have completed and signed the copyright transfer form and this has been included with the article submission 


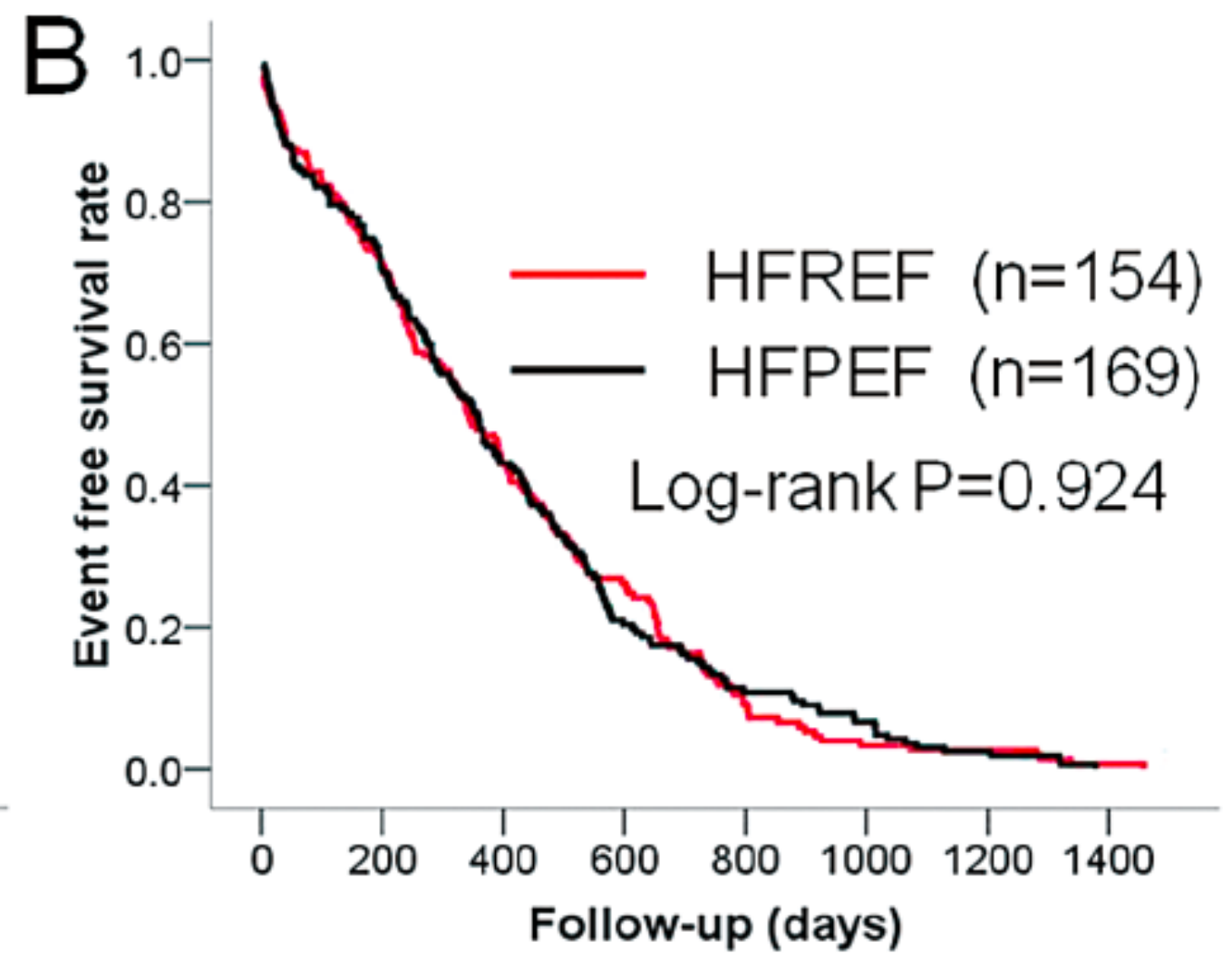

Figure 1. Kaplan-Meier curves for survival rate in heart failure with reduced ejection fraction (HFREF: red line, $n=154$ ) vs. HF and preserved EF (HFPEF: black line, $n=$ 169) [12]

Figure was published originally in:

Hamaguchi, S., et al., Mode of death in patients with heart failure and reduced vs. preserved ejection fraction: report from the registry of hospitalized heart failure patients. Circ J, 2012. 76(7): p. 1662-9. 


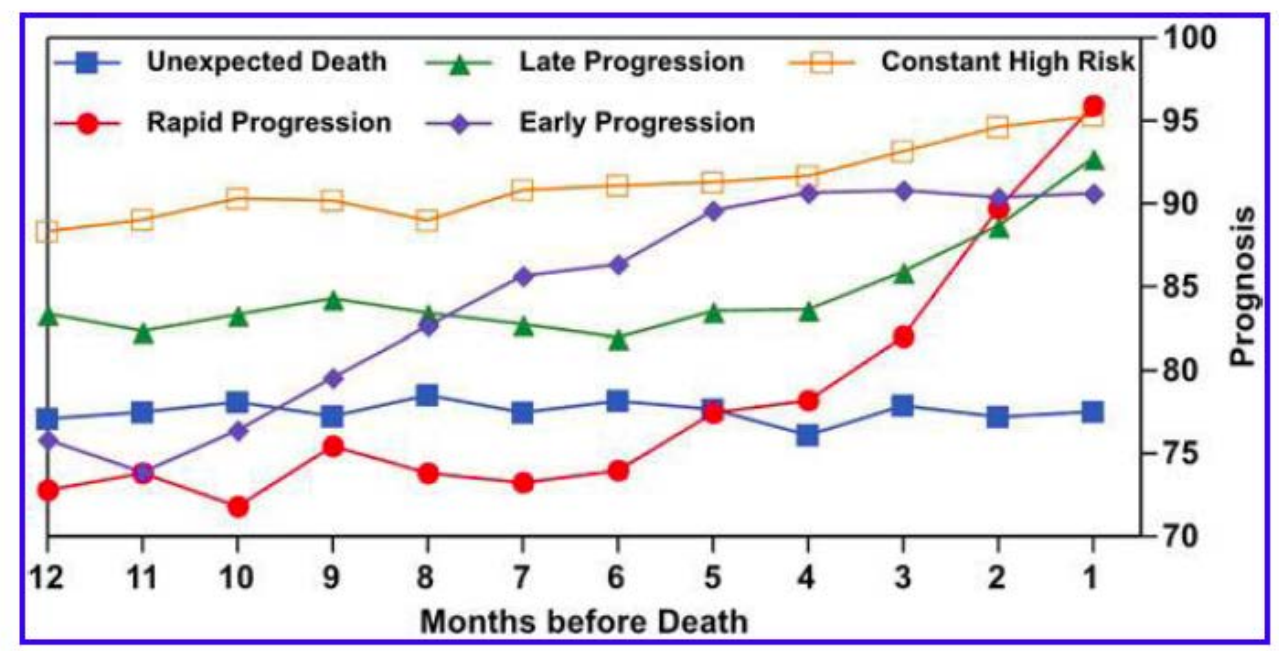

Figure 2. Trajectories of probability of death (prognosis) in HF patients based on retrospective analysis of cardiac and non-cardiac factors influencing the prognosis [14]

Figure was published originally in:

Kheirbek, R., et al., Trajectory of illness for patients with congestive heart failure. Journal of palliative medicine, 2013. 16(5): p. 478-484. 
Table 1. Clinical features of patients dying from systolic and diastolic HF (based on $[8,12])$

\begin{tabular}{|c|c|c|}
\hline \multirow[t]{2}{*}{ Feature } & \multicolumn{2}{|l|}{ Type of HF } \\
\hline & Systolic (HFREF) & Diastolic (HFPEF) \\
\hline Age (mean) & Younger (73.4 y) & Older (77.5 y) \\
\hline Sex (male \%) & $\begin{array}{l}\text { Predominantly male } \\
(70.8 \%)\end{array}$ & $\begin{array}{l}\text { More often female than in } \\
\text { the HFREF group ( } 45 \%)\end{array}$ \\
\hline \multicolumn{3}{|l|}{ General mortality } \\
\hline \multicolumn{3}{|l|}{ Cardiovascular deaths: } \\
\hline Progressive HF & $35-41 \%$ & $14 \%$ \\
\hline SCD & $40 \%$ & $27 \%$ \\
\hline Myocardial infarction & $7 \%$ & $3-5 \%$ \\
\hline $\begin{array}{l}\text { Non-cardiovascular } \\
\text { deaths }\end{array}$ & $15 \%-18 \%$ & $28-30 \%$ \\
\hline \multicolumn{3}{|l|}{ Cardiovascular deaths } \\
\hline SCD (\% of CVD) & $30-48 \%$ & $43 \%$ \\
\hline Progressive HF & $41-47.2 \%$ & $24 \%$ \\
\hline \multicolumn{3}{|l|}{$\begin{array}{l}\text { Non-cardiovascular } \\
\text { deaths }\end{array}$} \\
\hline Cancer (\% of non-CVD) & $44 \%$ & $33 \%$ \\
\hline Infection/Sepsis & $22 \%$ & $29 \%$ \\
\hline $\begin{array}{l}\text { Usual haemodynamic } \\
\text { profile of dying }\end{array}$ & $\begin{array}{l}\text { Low output } \\
\text { Low output }+ \text { congestion }\end{array}$ & $\begin{array}{l}\text { Congestion } \\
\text { Low output }+ \text { congestion }\end{array}$ \\
\hline
\end{tabular}

Note: HF = heart failure; HFREF = heart failure with reduced ejection fraction; HFPEF = heart failure with preserved ejection fraction; CVD = cardiovascular death; SCD $=$ sudden cardiac death. 
Table. 2 Description of haemodynamic variables

\begin{tabular}{|c|c|c|c|}
\hline Variable & Abbreviation & Unit & Definition \\
\hline $\begin{array}{l}\text { Left ventricle end- } \\
\text { diastolic volume }\end{array}$ & LVEDV & $\mathrm{ml}$ & $\begin{array}{l}\text { The volume of blood in left ventricle at } \\
\text { the end of diastole }\end{array}$ \\
\hline $\begin{array}{l}\text { Left ventricle end- } \\
\text { systolic volume }\end{array}$ & LVESV & $\mathrm{ml}$ & $\begin{array}{l}\text { The volume of blood in left ventricle at } \\
\text { the end of systole }\end{array}$ \\
\hline Stroke volume & SV & $\mathrm{ml}$ & $\begin{array}{l}\text { The volume of blood pumped from one } \\
\text { ventricle with one contraction }\end{array}$ \\
\hline Cardiac output & $\mathrm{CO}$ & $\mathrm{l} / \mathrm{min}$ & $\begin{array}{l}\text { The volume of blood pumped from one } \\
\text { ventricle in one minute: } \mathrm{CO}=\mathrm{SV} \bullet \text { heart } \\
\text { rate }\end{array}$ \\
\hline Ejection fraction & $\mathrm{EF}$ & $\%$ & $\begin{array}{l}\text { The volumetric fraction of blood } \\
\text { pumped from one ventricle with each } \\
\text { contraction } \\
\mathrm{EF}=\mathrm{SV} / \mathrm{LVEDV} \bullet 100 \% \text { or } \\
\mathrm{EF}=(\mathrm{LVEDV}-\mathrm{LVESV}) / \mathrm{LVEDV} \\
100 \%\end{array}$ \\
\hline
\end{tabular}

